\title{
Model of location for service station
}

\author{
J. de Rezende ${ }^{1} \&$ N. F. F. Ebecken ${ }^{2}$ \\ ${ }^{1}$ PETROBRAS, Brazilian Energy Company \\ ${ }^{2}$ COPPE/Federal University of Rio de Janeiro, Brazil
}

\begin{abstract}
This paper presents a process for supporting the decision when selecting the best location to evaluate a service station. In accordance with experts, the three main factors responsible for the success of business are: the mix of products, when one tries to offer the right product for the right person in the right place; the business management in which method and tools are included; and the business location. The selection of a good location is associated with a high number of variables; some of which can be intangible, inexact, uncertain and subjective. Therefore, we have chosen to use techniques of Fuzzy Logic for the construction of a location hierarchical model which considered the importance of different factors, in a differentiated form, according to the type of service station which was evaluated. Through the application of quality and quantitative research, the analysis of the results, and the deep experience of specialists, it was possible to identify and quantify the main location factors utilized in the model.
\end{abstract}

Keywords: hierarchal model; fuzzy model; correspondence analysis; service station location.

\section{Introduction}

The objective of this article is to develop a model of evaluation of the service station (SS) in Argentina based on characteristics of location, developing a methodology that enables the identification of factors that influence in the location of service stations, permitting better evaluation of cover strategies, expansion and shrinkage. The proposed methodology should include connected aspects to the profile of the business, evaluate the potential of business of the existing service stations, analyzing results based on market potential and identifying service stations with deficit, but with big market potential. We can 
classify and analyze the service stations according to the location index and its real invoicing, in 4 quadrants:

- Quadrant I: SS with low invoicing and high location index. This SS needs to be better worked, since they possess potential to improve their invoicing.

- Quadrant II: SS with invoicing and high location index. This SS will serve as reference for the others.

- Quadrant III: SS with high invoicing and low location index. This SS needs to be better studied, since their unidentified characteristics contribute to high invoicing and that will be incorporated to the model.

- Quadrant IV: SS with invoicing and low location index. This SS can be discarded.

The hypotheses that will be tested are focused on the importance of location in the success of an undertaking and in the importance of the different factors that influence, in different ways, the diverse kinds and characteristics of a service station which fit into the economic, social, and geographical aspects. In this context we are going to consider four kinds of service stations, which are:

- Urban stations: located in urban perimeter, mini-markets serving quick meals and with representative invoicing;

- Urban VIP stations: serving high-class society, with modern image, products of high value, mini-markets as convenience stores, located in high income areas or with high representation in opinionated areas;

- Driveway stations: located in highways, occupying big areas, with big parking stations and intensive use of diesel.

- Suburban stations: located in urban areas near the exit or entrance of highways, focusing on low value products and intensive use of diesel and CNG;

\section{Fuel market}

The main companies of the sector make painstaking pleas in offering their service through modern, spacious, attractive and functional service stations, which today already are considered barely a mini-market. It is worth highlighting that we understand service stations like: the responsible gas station by the sales of fuel through its gas pumps, the convenience stores responsible for the sales of several edible and entertaining products, the center responsible for lubrication through oil exchange, and other services that will be available to better serve its clients, such as pharmacy, mechanic offices, video rental agency, 24 hour banks, etc, all situated in common areas.

Beyond the quality of the products, the quality of the service given, the ease of payment and of the big influence exercised through the main points, one of the big challenges is to find the best adequate places for installation of the new service stations, minimizing installation investments, and maximizing the return expected of that operation.

The relation between the oil company and the management of the service stations come in different forms: 
- Company Owned Company Operated (COCO) - when the petroleum company is the holder and responsible for the management of the service station;

- Company Owned Dealer Operated (CODO) - when the petroleum company is holder of the service station, however outsource management for other companies to operate;

- Dealer Owned Dealer Operated (DODO) - when the company user is holder of the service station and responsible for its own management.

\subsection{Argentinean distribution market}

In December of 2002 it had 6.283 service stations, $71 \%$ are of private firms which operate in the market through a contractual relation with some flag (DODO), 20\% don't have a bond with any flag and because of this are considered "White" and the remainder (9\%) are property of their own tankers (COCO or CODO). The location of the service stations show high concentration in the Province of Buenos Aires (40\%), followed by Santa Fé (12\%), Córdoba (11\%) and Federal Capital (7\%). The remaining 30\% are distributed through the provinces of the country.
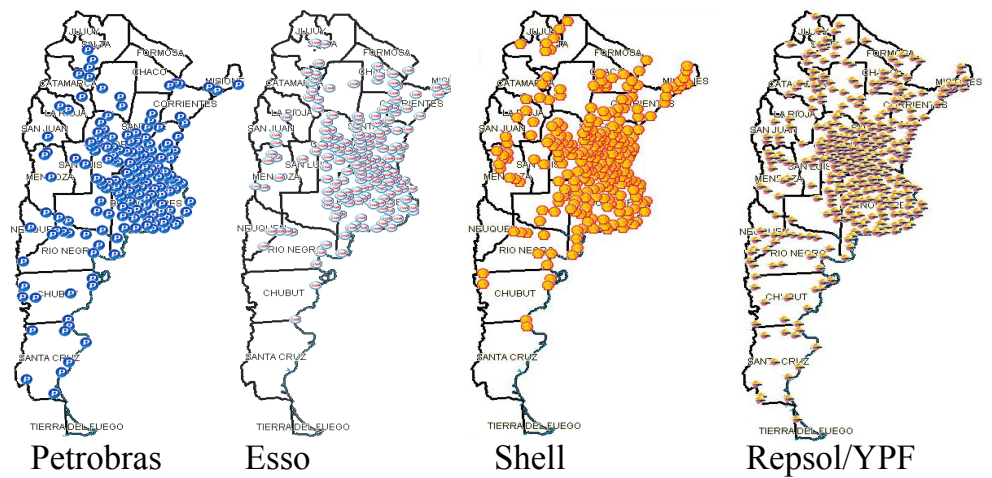

Figure 1: Concentration of service station. Source: Interchange with the company.

The service stations market in December of 2004 was composed of 5.967 service stations, having had a diminution around December of 2002 of approximately 5\%. The Argentinean fuel market is broadly dominated by the Spanish Repsol-YPF, with around 50,8\% of market share of the sale of fuel, followed by Shell with 16,0\%, Petrobras with 14,2\%, Esso with 13,8\% and the remaining with 5,2\%, given those of December of 2004 .

\subsection{Qualitative research}

The strategy of qualitative research consists of an assembly of interviews focused on Holders, Users and Managers of service stations and that had like a base a questionnaire previously concocted. 
As result we observed the importance of the invoicing of the mini-market for the service station and the growing importance that the User gives to the minimarket. We also establish that, for still being an incipient business for the big majority of the interviewed, the management "professional" of the mini-market still leaves much to desire, being necessary, on the occasion of his implementation, a program of specific managerial training.

\subsection{Quantitative research}

The strategy of quantitative research consists in the application of a questionnaire, with emphasis on a Sub-Stratified Sample Plan, considering the geographical variables and the type of management of the service stations. There were a total of 514 service stations researched. The rate of return of the questionnaires was about $72 \%$, which we judge broadly satisfactory. A research on screen was carried out between the months of May and June of 2005. The construction of the questionnaire applied based itself on a qualitative research previously carried out which gave us an idea of some verified problems. The application of the questionnaires was opted by Commercial Representatives, where the same were requested to be applied through an interview with the representatives of the service stations. The research was carried out on the basis of a questionnaire with 101 questions, being that some were open and others closed, approaching aspects of identification and characteristics of the service stations, road service, mini-market service, and other services. There were 136 variables researched characterized like continuous numerical variables, discrete numerical variables and categorical variables.

\section{Analysis of facts through varied approach analysis method}

The handling of the facts obtained in the quantitative research, through the Correspondence Analysis, part of a matrix with 514 records, 23 variables and 90 categories. As stop criteria in the analysis, relative level of contribution of the factors was utilized, and we concluded that the first three factors would be sufficient to describe the main characteristics of the service stations, being that the cut is made from the third to fourth factor. In theory, the factors 4 to 7 would be significant, but the analysis of the contribution of the variables to the factors show that they are merely repetitions already obtained on factors 1 to 3 .

\subsection{Conclusions of data analysis}

Through the techniques of data analysis, more precisely correspondence analysis we form the 4 (four) service station groups: The "Urban" service stations are associated with the following characteristics: "Area of the small mini-market", "weak commercialization of diesel", "weak invoicing of fast food", "predominant flow of traffic from the neighborhood to the downtown area", "small road area", "good access to the SS", "good visibility to the SS", 
"predominant direction from home to work", "good invoicing of CNG" and "visibility of the SS for the client facing the flow of circulation". The "Urban VIP" service stations are associated to the following characteristics: "large minimarket area", "predominant flow of traffic from the downtown area to the neighborhood", "excellent CNG invoicing", "excellent invoicing of nafta super", "dual SS", "excellent visibility of the SS", "excellent access to the SS", "minimarket with fast food", "very large road area", "predominant direction from work to home", "very good invoicing of nafta", "invoicing of weak fast food", "invoicing of regular diesel", "good invoicing of CNG", "profile of the social class dominating medium high", "excellent invoicing of the mini-market" and "regular invoicing of fast food". The "Driveway" service stations are associated to the following characteristics: "flow of traffic predominant in highways", "large total area", "large road area", "half block visibility for the client", "excellent invoicing of diesel" and "excellent invoicing of roads".

The "Suburban" service stations are associated to the following characteristics: "Visibility of the SS for the client near the circulatory", "excellent ticket medium of the mini-market", "visibility of the SS for other clients", "predominant meaningless direction" and "excellent invoicing of diesel"'.

\subsection{Theory of the applied location to the service stations}

The main factors of good location are in the visibility and in the accessibility, which signifies that you want to locate your shop where people can see and have easy access. For maximum visibility, consider an area where the clients can see you at a possible distance needed to have time to decide to go to your store. And for excellent access, signifies a broad, easy access, with clear entrances and exits in both directions of traffic.

Associated with these factors are also the dynamic aspects of the consumer behavior, the importance of regional aspects in the demand of the mix of product, going beyond the quality and quickness of the service.

\subsection{Fuzzy methodology applied to the location}

In our particular case, the decision-making related to the selection of the location of the service station is associated to an elevated number of variables to be considered, being that some are intangible, others inexact, uncertain and subjective. Associated with these variables exists a personal perception of the decision maker about the relative importance of each one of them in the decision making process. The study consists of identifying and setting out socioeconomic variables, ways of generating hierarchal options arranged for the location of the service stations, considering several profiles, by which motive we opt the utilization of the methodology based on the Fuzzy logic.

In the case of the offering of factors, the measure to be adopted in this study will attribute four values to the units of location of Argentinean territory: 
- Level A, EXCELLENT, when the territorial offering will be representing situations where the intensity with which that factor occurs is superior to the requested.

- Level B, GOOD, when the territorial offering will be representing situations where the intensity with which that the factor occurs is compatible with the requested.

- Level C, REGULAR, when the territorial offering will be representing situations where the intensity with which that factor occurs is lower than the requested.

- Level D, WEAK, when the territorial offering will be representing situations where the intensity with which that factor occurs is insignificant to the requested.

In the study of the demand of factors, it is necessary to consider the typology as an assembly of common specific characteristics to a service station project. The measure of demand will adopt four values:

- Level A, CRITICAL, when the demand of an activity will be representing situations where the necessity of a factor present in high level is crucial to the success of the undertaking.

- Level B, CONDITIONING, when the demand of an activity will be representing situations where the intensity of the demand by a factor is conditioned in such a way that, if not found in satisfactory quantities, will have some additional cost for the maintenance of the undertaking.

- Level C, LITTLE CONDITIONING, when the demand of an activity will be representing situations where the intensity of the demand of a factor is little conditioned, in such a way that if not found in satisfactory quantities, the undertaking continues viable, but subject to additional costs and critical periods.

- Level D, IRRELEVANT, when the demand of an activity will be representing situations where the intensity of the demand by a factor is irrelevant, in such a way that if not found, there will be little difference toward the success or failure of the undertaking.

The result of the processing between the matrices of offering and demand is translated into a new matrix, one of possibilities of location (location $x$ typologies), that shows the rank of adaptation of typology of each kind of service station for each region or specific location. This rank obtained establishes a "ranking", expressed in function of convergence for the numerical value " 1 ". Values larger than " 1 " indicates an excess of offering, smaller values indicate that at least one factor of location was left from being attended satisfactorily. The arrangement of matrices also permits a verification of performance by locality or activity.

\subsection{Theoretical conception of the hierarchal location model [19]}

The model confronts, initially, the situations of demand of the activity to be located (SS) with those of territorial offering of general factors, and uses the Matrix of pertinence relations below. 


\begin{tabular}{|c|l|l|l|l|}
\hline & \multicolumn{1}{|c|}{ Weak } & Regular & Good & Excellent \\
\hline IR & 1 & $1+\mu_{B_{1}}(x) / n$ & $1+\mu_{B_{2}}(x) / n$ & $1+\mu_{B_{3}}(x) / n$ \\
\hline LC & $\mu_{B_{1}}(x) / \mu_{A_{2}}(x)$ & 1 & $1+\mu_{B_{1}}(x) / n$ & $1+\mu_{B_{2}}(x) / n$ \\
\hline CO & $\mu_{B_{1}}(x) / \mu_{A_{3}}(x)$ & $\mu_{B_{2}}(x) / \mu_{A_{3}}(x)$ & 1 & $1+\mu_{B_{1}}(x) / n$ \\
\hline CR & $\mu_{B_{1}}(x) / \mu_{A_{4}}(x)$ & $\mu_{B_{2}}(x) / \mu_{A_{4}}(x)$ & $\mu_{B_{3}}(x) / \mu_{A_{4}}(x)$ & 1 \\
\hline
\end{tabular}

Where, $\mathrm{n}$ is like the number of factors considered in the operation.

In different applications, like the ones already demonstrated, the model can be generalized in several ways without losing its basic characteristics. This way, different levels are permitted for the same attribute when confronted with the different requirements. The model also can and should be customized according to the area of study.

The implementation of the model was given through real facts collected in the quantitative and qualitative researches, beyond facts of social and demographic studies. In the application of the model, we opted to randomly choose service stations that satisfy basic requirements, in what refers to the necessary information toward its implementation.

It is worth highlighting that the service stations were evaluated according to its type, being, Urban, Urban VIP, Driveway and Suburban, since inside each kind exists a larger homogenization, including the regional aspects of each, and the characteristics of business included road service, mini-markets and other services.

\subsection{Profile of demand of each kind of service station}

Sixteen (16) factors were considered classified by specialists according to their rank of importance for each kind of service station. The factors chosen were: Accessibility, Visibility, Road Position, ES area, road area, mini-market area, flow direction, predominant direction, social class, kind of product, rate of vehicle/habitant, number of vacancies, competition, medium speed, generators of traffic and demographic density. It is worth highlighting that other important location factors did not enter in the model because of absolute absence of facts or information.

\subsection{Profile of offering of the Urban service station}

The same methodology used to classify factors of the matrix of demand was utilized for the classification of factors of the matrix of offering. Twenty (20) service stations were randomly selected of the Urban kind, representing approximately $10 \%$ of the stations of that kind, through the method of Systematic Sample. 
In the analysis of the facts a positive correlation of the order of 0.6881 was verified between the variables Location Indexes and the invoicing of the stations researched. This value can be considered very satisfactory, since we know that invoicing is influenced also through the mix of products and by the kind of management. Through the following graphic, we can visualize the stations researched according to their invoicing and location index distributed as axes corresponding to $\mathrm{X}$ and $\mathrm{Y}$, respectively. In the graphic analysis we verify that the stations belonging to the second quadrant should serve as reference, mainly for the stations belonging to the first quadrant, since the same, although possessing a Location Index above the average, invoicing did not correspond to its potential. These stations should be better evaluated in what concerns to management and to the mix of products offered.

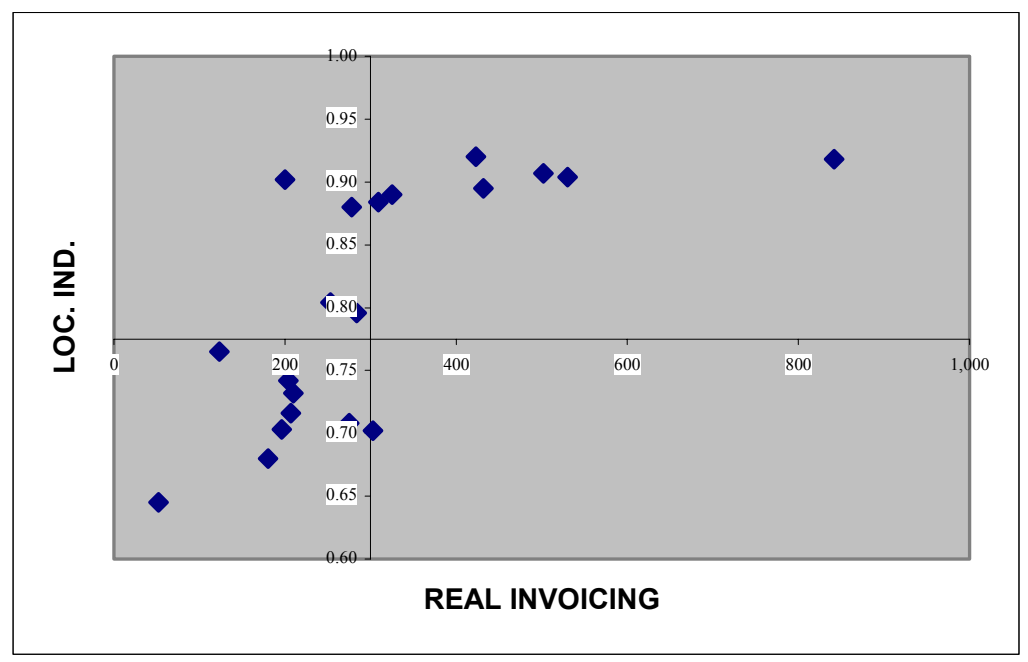

Figure 2: $\quad$ Real invoice x location index: ES Urban.

\subsection{Profile of offering of the Urban VIP service station}

In relation to the small number of service stations of this kind, those which arranged the necessary information were selected or those without any missing information in the factors were selected. We clarify that the big majority of those stations classify themselves as the kind of operation COCO. In the analysis of the facts, we verify a positive lineal correlation in the order of 0,7595 between the location index variables and the invoicing of the stations researched. We consider this value satisfactory enough in regards to the previous explanation.

\subsection{Profile of offering of the driveway service station}

Twenty-six (26) service stations were randomly selected of this kind, through the method of Systematic Sample. 
In the analysis of facts, we verify a positive linear correlation in the order of 0,7923 between the variables Location Indexes and the invoicing of the stations researched. We consider this value satisfactory enough in regards to previous explanation.

\subsection{Profile of offering of the suburban service station}

Twenty-one (21) service stations were randomly selected of this kind, through the method of Systematic Sample. In the analysis of the facts, we verified a positive linear correlation in the order of 0,7482 between the variables Location Indexes and the invoicing of the stations researched. We consider this value satisfactory enough in regards to the previous explanation.

\section{Conclusions}

The main motivation of the article was to develop a method of evaluation of service stations based on characteristics of location. This methodology is supported by a hierarchal location model that considered the importance of different factors in different ways, according to the kind of service station evaluated. Through the application of the quantitative and qualitative researches, the analyses of results and of the deep experience of the specialists, it was possible to identify and quantify the main location factors utilized in the model.

The hypotheses that were tested relating to the importance of location and the different location factors that influence the diverse kinds of service stations in different ways were fully accepted.

We establish that the factors have differentiated importance according to the kind of service station or any undertaking.

Also, according to the analyses of the results obtained between the invoicing and the location index of the four kinds of service stations, we verify that the correlation between them was of 0,6881 for the Urban, 0,7595 for the Urban VIP, 0,7923 for the Driveway and 0,7482 for the Suburban. The meaning of those numbers reflects directly in the importance of the location index in the invoicing of the service stations. If we admit a linear relation between those variables, what by fact demonstrates the coefficient of correlation in the sample, through a simple linear model, we will have an explanation of the model varying $44,35 \%$ for the Urban stations, 55,98\% for the Suburban Stations, 57,68\% for the Urban VIP, reaching $62,77 \%$ for the Driveway Stations. In other words, this means that a location is responsible at least for $45 \%$ of the invoicing and can reach almost $63 \%$.

We also establish that the invoicing of the mini-market contributes between 7 and $12 \%$, depending on the type of service station, with the invoicing of the service station, and that a positive linear dependence exists between the invoicing of roads and mini-markets.

The model utilized has as a main characteristic the possibility of being customized for different localities or regions. Therefore, it is fundamental that this customization is preceded of field work and has the guarantee of the specialists of the business of the region to be applied. On the other hand, we 
must not keep in mind only the present, but take into consideration the possible future alterations of the environment, the motivation or need of purchase that is going to be attended, which characterizes strong dynamic in the factors as in its valorization.

\section{References}

[1] Birkin, M. Retail Geography and Intelligent Network Planning. John Wiley and Sons Ltd, 2002.

[2] Buckley, M. P. Location strategies: Na overbuilt retail market. Retail Merchandiser, vol 33, New Yorker, 1993.

[3] Curry, B., Moutinho, L. Computer models for site location decision. International Journal of Retail \& Distribution Management, Vol 20. Bradford, 1992.

[4] Dell'Acqua, G. Highway accidents analysis using Fuzzy pattern recognition. University of Naples Federico II, Italy, 2001.

[5] England, J. Retail Impact Assessment: A Guide to Best Practice. Taylor \& Francis Books Ltd, 2000.

[6] Hernandez, T., Bennison, D. The art and science of retail location decision. International Journal of Retail \& Distribution Management, Vol 28. Bradford, 2000.

[7] Kahraman, C., Dogan, I. Fuzzy Group decision-making for facility location selection, Information Sciences 157, University of California, Berkeley, California, USA, 2003.

[8] Kerber, R. Discretization for numeric attributes. $10^{\text {th }}$ National Conference on Artificial Intelligence. USA, 1992.

[9] Kuo, R. J., Chi, S. C., Kao, S. S. A decision support system for locating convenience store through fuzzy AHP. Computers \& Industrial Engineering 37. Elsevier Science Ltd, 1999.

[10] Kuo, R. J., Chi, S. C., Kao, S. S. A decision support system for selecting convenience store location through integration of fuzzy AHP and artificial neural network. Computers in Industry 47. Elsevier Science B. V., 2002.

[11] Liu, B., Hsu, W., Chen, S., Ma, Y. Integrating Classification and Association Rule Mining. New York: KDD-98, 1998.

[12] McGoldrick, P. Retail Marketing. McGraw-Hill Europe, 2000.

[13] Narasimhan, Ram. A fuzzy subset characterization of a site-selection problem. Decision Sciences, Vol. 10, 1979.

[14] Punj, G., Stewart, D. W. Cluster analysis in marketing research: Review and suggestions for applications. Journal of Marketing Research, 1983.

[15] Reynolds, J., Cuthbertson, C. Retail Strategy: The view from the bridge. London: Elsevier Butterworth-Heinemann, 2004.

[16] Roger, D. Retail Location Analysis in Practice. Oxford Reports on Retailing. University of Oxford, 1992.

[17] Salvaneschi, L., Location, location, location : How to select the best site for your business. Oregon: Oasis Press-PSI Research, 2002.

[18] Templeton College University of Oxford. Retail Location Analysis, 2004. 
[19] Toledo, O. M., Cosenza, C. A. N. The evolution of the COPPETECCOSENZA Model of Industrial Location for the Hierarchal Fuzzy Model and its Application. COPPE/UFRJ, 2002. ( In portuguese ) 